\title{
छs
}

\section{Spin-Orbit Coupled Degenerate Fermi Gases}

\author{
Pengjun Wang, ${ }^{1}$ Zeng-Qiang Yu, ${ }^{2}$ Zhengkun Fu, ${ }^{1}$ Jiao Miao, ${ }^{2}$ Lianghui Huang, ${ }^{1}$ \\ Shijie Chai, ${ }^{1}$ Hui Zhai, ${ }^{2, *}$ and Jing Zhang ${ }^{1, \dagger}$ \\ ${ }^{1}$ State Key Laboratory of Quantum Optics and Quantum Optics Devices, Institute of Opto-Electronics, Shanxi University, \\ Taiyuan 030006, People's Republic of China \\ ${ }^{2}$ Institute for Advanced Study, Tsinghua University, Beijing, 100084, People's Republic of China
}

(Received 30 May 2012; published 27 August 2012)

In this Letter, we report the first experimental realization and investigation of a spin-orbit coupled Fermi gas. Both spin dephasing in spin dynamics and momentum distribution asymmetry of the equilibrium state are observed as hallmarks of spin-orbit coupling in a Fermi gas. The single particle dispersion is mapped out by using momentum-resolved radio-frequency spectroscopy. From momentum distribution and momentum-resolved radio-frequency spectroscopy, we observe the change of fermion population in different helicity branches consistent with a finite temperature calculation, which indicates that a Lifshitz transition of the Fermi surface topology change can be found by further cooling the system.

In the past decade, quantum simulations with ultracold atoms have investigated many fascinating quantum phenomena in a highly controllable and tunable way. However, until recently, an important interaction has not been explored in cold atomic gases, that is, the spin-orbit (SO) coupling. Last year, a pioneering experiment from National Institute of Standards and Technology first realized a SO coupled Bose-Einstein condensate using the twophoton Raman process [1], which will give rise to new quantum phases [2-5]. In real materials, SO coupling plays an important role in many physical systems over a wide range of energy scales, from determining the electron structure inside an atom to giving birth to topological insulators in solid state materials [6,7]. Since all these systems are fermionic, from the viewpoint of quantum simulation it is desirable to experimentally realize SO coupled degenerate Fermi gases. The physical effects of SO coupling in a degenerate Fermi gas will be quite different from those in a Bose system.

In this Letter, we report the experimental realization of a SO coupled degenerate Fermi gas, and the main results include the following: (i) starting from a fully spin polarized state and turning on the Raman coupling, we have observed SO coupling induced spin dephasing in the quantum spin dynamics; (ii) for equilibrium states we have observed the asymmetry in the spin-resolved momentum distribution due to SO coupling; (iii) from momentum distribution we have observed the change of fermion population in different helicity branches as the density of the fermion increases; and, finally, (iv) we have used momentum-resolved radio-frequency (rf) spectroscopy to map out single particle dispersion and confirmed the change of population in the helicity branch. The change of population is consistent with a finite temperature theoretical calculation, which indicates that a sharper Lifshitz transition of changing the Fermi surface topology can be found by further cooling the system. This progress will enable us to study many interesting phenomena predicated for SO coupled atomic Fermi gases in the future [8-14].

In our experiment, a degenerate Fermi gas of $2 \times 10^{6}$

${ }^{40} \mathrm{~K}$ atoms in the lowest hyperfine $|9 / 2,9 / 2\rangle$ state is first prepared in an optical dipole trap. The optical dipole trap is composed of two horizontal crossed beams of $1064 \mathrm{~nm}$ at $90^{\circ}$ along the $\hat{x} \pm \hat{y}$ direction overlapped at the focus, as shown in Fig. 1(a). The temperature of the Fermi gas is about $0.3-0.4 T_{\mathrm{F}}\left(T_{\mathrm{F}}\right.$ is the Fermi temperature) when the trap frequency reaches $2 \pi \times(116,116,164) \mathrm{Hz}$ along the $(\hat{x}, \hat{y}, \hat{z})$ direction [15]. A pair of Helmholtz coils provides a homogeneous bias magnetic field along $\hat{y}$ (quantization axis), which is precisely controlled by a carefully designed scheme described in Ref. [16] to reduce the magnetic field drift and the magnetic noise.

The method we used to generate SO coupling is the same as reported by the National Institute of Standards and Technology group for the ${ }^{87} \mathrm{Rb}$ Bose condensate [1]. In the ${ }^{40} \mathrm{~K}$ system, two spin- $1 / 2$ states are chosen as two magnetic sublevels $|\uparrow\rangle=|9 / 2,9 / 2\rangle$ and $|\downarrow\rangle=|9 / 2,7 / 2\rangle$. They are coupled by a pair of Raman beams with the wavelength $\lambda=773 \mathrm{~nm}$, the frequency difference $\omega$, and the coupling strength $\Omega$. Two Raman lasers counterpropagate along the $\hat{x}$ axis and are linearly polarized along the $\hat{y}$ and $\hat{z}$ directions, respectively, corresponding to $\pi$ and $\sigma$ polarization relative to quantization axis $\hat{y}$ [as shown in Fig. 1(a)]. The recoil momentum $k_{r}=k_{0} \sin (\theta / 2)$ and recoil energy $E_{r}=k_{r}^{2} / 2 m=h \times 8.34 \mathrm{kHz}$ are taken as natural momentum and energy units, where $k_{0}=2 \hbar \pi / \lambda$ and $\theta=180^{\circ}$ is the angle between two Raman beams. A Zeeman shift $\omega_{\mathrm{Z}} / 2 \pi=10.27 \mathrm{MHz}$ between these two magnetic sublevels is produced by the homogeneous bias magnetic field at $31 \mathrm{G}$. When the Raman coupling is at resonance [at $\omega / 2 \pi=10.27 \mathrm{MHz}$ and two-photon Raman detuning $\left.\delta=\hbar\left(\omega_{\mathrm{Z}}-\omega\right) \approx 0\right]$, the detuning 

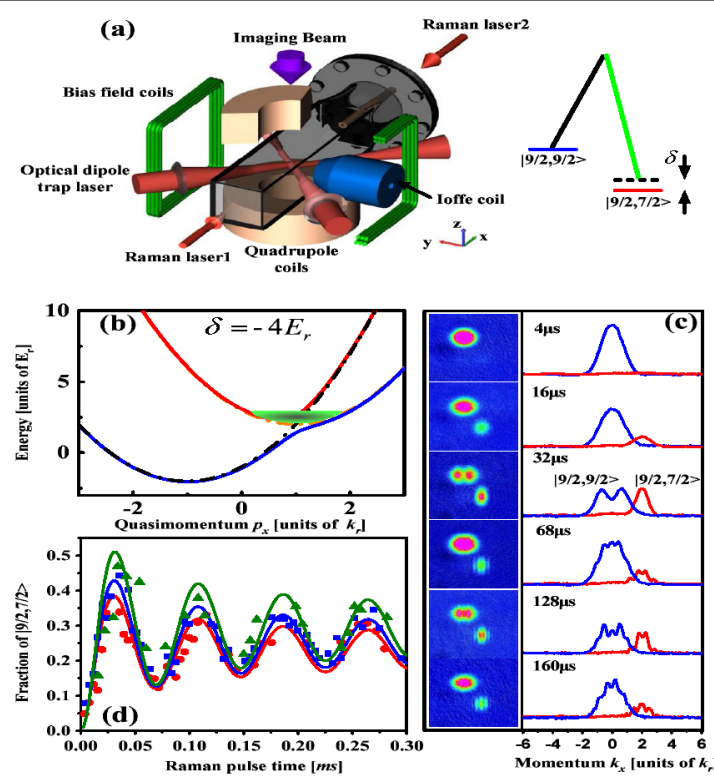

FIG. 1 (color online). Experimental setup and Raman-induced quantum spin dynamics. (a) Schematic of the experimental setup and the Raman coupling of two hyperfine levels of ${ }^{40} \mathrm{~K}$. (b) The energy dispersion with $\delta=-4 E_{r}$. The system is initially prepared with all atoms in the $|9 / 2,9 / 2\rangle$ state. (c) Time-of-flight image (left) and integrated time-of-flight image (integrated along $\hat{y})$ at different duration times for $|\uparrow\rangle$ (blue) and $|\downarrow\rangle$ (red). The parameters are $k_{\mathrm{F}}=1.35 k_{r}$ and $T / T_{\mathrm{F}}=0.35$. (d) The population in $|9 / 2,7 / 2\rangle$ as a function of duration time of the Raman pulse. $k_{\mathrm{F}}=1.9 k_{r}$ and $T / T_{\mathrm{F}}=0.30$ for red circles, $k_{\mathrm{F}}=1.35 k_{r}$ and $T / T_{\mathrm{F}}=0.35$ for blue squares, and $k_{\mathrm{F}}=1.1 k_{r}$ and $T / T_{\mathrm{F}}=$ 0.29 for green triangles. The solid lines are theory curves with $\Omega=1.52 E_{r}$.

between $|9 / 2,7 / 2\rangle$ and other magnetic sublevels like $|9 / 2,5 / 2\rangle$ is about $h \times 170 \mathrm{kHz}$, which is 1 order of magnitude larger than the Fermi energy. Thus we can safely disregard other levels and treat this system as a spin- $1 / 2$ system. The same as in the boson experiment, this scheme generates an effective single particle Hamiltonian as [1]

$\mathcal{H}=\left(\begin{array}{cc}\frac{1}{2 m}\left(\mathbf{p}-k_{\mathrm{r}} \hat{\mathbf{e}}_{x}\right)^{2}-\frac{\delta}{2} & \frac{\Omega}{2} \\ \frac{\Omega}{2} & \frac{1}{2 m}\left(\mathbf{p}+k_{\mathrm{r}} \hat{\mathbf{e}}_{x}\right)^{2}+\frac{\delta}{2}\end{array}\right)$.

Here, p denotes the quasimomentum of atoms, which relates to the real momentum $\mathbf{k}$ as $\mathbf{k}=\mathbf{p} \mp k_{r} \hat{\mathbf{e}}_{x}$ with $\mp$ for spin-up and -down, respectively. This Hamiltonian can be interpreted as an equal weight combination of Rashbatype and Dresselhaus-type SO coupling [1]. Finally, before time-of-flight measurement, the Raman beams, the optical dipole trap, and the homogeneous bias magnetic field are turned off abruptly at the same time, and a magnetic field gradient along the $\hat{y}$ direction provided by an Ioffe coil is turned on. Two spin states are separated along the $\hat{y}$ direction, and imaging of atoms along the $\hat{z}$ direction after $12 \mathrm{~ms}$ expansion gives the momentum distribution for each spin component.

Spin dynamics.-We first study the Rabi oscillation between the two spin states induced by the Raman coupling. All atoms are initially prepared in the $|\uparrow\rangle$ state. The homogeneous bias magnetic field is ramped to a certain value so that $\delta=-4 E_{r}$; that is, the $\mathbf{k}=0$ component of state $|\uparrow\rangle$ is at resonance with the $\mathbf{k}=2 k_{r} \hat{\mathbf{e}}_{x}$ state of the $|\downarrow\rangle$ component, as shown in Fig. 1(b). Then we apply a Raman pulse to the system and measure the spin population for different duration times of the Raman pulse. A similar experiment in the boson system yields an undamped and completely periodic oscillation, which can be well described by a sinusoidal function with frequency $\Omega$ [17]. This is because, for bosons, a macroscopic number of atoms occupy the resonant $\mathbf{k}=0$ mode, and therefore there is a single Rabi frequency determined by the Raman coupling only, while, for fermions, atoms occupy different momentum states. Precisely due to the effect of SO coupling, the coupling between the two spin states and the resulting energy splitting are momentum dependent, and atoms in different momentum states oscillate with different frequencies [as shown explicitly in Eq. (2) later]. Hence, dephasing naturally occurs and the oscillation will be inevitably damped after several oscillation periods. In our case, the spin-dependent momentum distribution shown in Fig. 1(c) clearly shows the out-of-phase oscillation for different momentum states.

To determine the value of $\Omega$ from the measurements, we fix Raman coupling and vary atom density by changing the total number of fermions or the trapping frequency, and we obtain several different oscillation curves, as shown in Fig. 1(d). Then we fit them to the theory with a single fitting parameter $\Omega$. Theoretically, for a noninteracting system, the population of the $|\downarrow\rangle$ component is given by

$n_{\downarrow}\left(\mathbf{k}+2 k_{r} \hat{\mathbf{e}}_{x}, \mathbf{r}, t\right)=n_{\uparrow}(\mathbf{k}, \mathbf{r}, 0) \frac{\sin ^{2} \sqrt{\left(k_{x} k_{r} / m\right)^{2}+\Omega^{2} / 4} t}{1+\left(\frac{2 k_{x} k_{r}}{\Omega m}\right)^{2}}$,

where $t$ is the duration time of the Raman pulse, $n_{\uparrow}(\mathbf{k}, \mathbf{r}, 0)$ is the equilibrium distribution of the initial state in the local density approximation, and the temperature of the initial cloud is determined by fitting the time-of-flight image to the momentum distribution of free fermions in a harmonic trap. From Eq. (2), one can see that the momentum distribution along the $\hat{x}$ direction of the $|\downarrow\rangle$ component is always symmetric with respect to $2 k_{r}$ at any time, and the experimental data show that this is indeed the case, as shown in Fig. 1(c). The theoretical expectation of the total population in the $|\downarrow\rangle$ component is given by $N_{\downarrow}(t)=$ $\int d^{3} \mathbf{k} d^{3} \mathbf{r} n_{\downarrow}(\mathbf{k}, \mathbf{r}, t)$, and in Fig. 1(d), one can see that there is an excellent agreement between the experiment data and theory, from which we determine $\Omega=1.52(5) E_{r}$. Since our current experiment is performed in the weakly interacting regime with $s$-wave scattering length $a_{s}=169 a_{0}$, 
we have verified that the interaction effect is negligible [15].

Momentum distribution.-We focus on the case with $\delta=0$ and study the momentum distribution in the equilibrium state. We first transfer half of ${ }^{40} \mathrm{~K}$ atoms from $|\downarrow\rangle$ to $|\uparrow\rangle$ by using a radio-frequency sweep within $100 \mathrm{~ms}$. Then the Raman coupling strength is ramped up adiabatically in $100 \mathrm{~ms}$ from zero to its final value, and the system is held for another $50 \mathrm{~ms}$ before time-of-flight measurement. We have also varied the holding time and find that the momentum distribution does not change; thus, we conclude that the system has reached equilibrium in the presence of SO coupling. Since SO coupling breaks spatial reflectional symmetry $\left(x \rightarrow-x\right.$ and $\left.k_{x} \rightarrow-k_{x}\right)$, the momentum distribution for each spin component will be asymmetric, i.e., $n_{\sigma}(\mathbf{k}) \neq n_{\sigma}(-\mathbf{k})$, with $\sigma=\uparrow, \downarrow$. On the other hand, when $\delta=0$ the system still satisfies $n_{\uparrow}(\mathbf{k})=n_{\downarrow}(-\mathbf{k})$. The asymmetry can be clearly seen in the spin-resolved time-offlight images and integrated distributions displayed in Figs. 2(a) and 2(b), where the fermion density is relatively low, while it becomes less significant when the fermion density becomes higher, as shown in Fig. 2(c), because the strength of SO coupling is relatively weaker compared to the Fermi energy. In Figs. 2(a)-2(c), the integrated momentum distribution is fitted by the theoretical calculation to determine the temperature and the chemical potential at the center of the trap [15]. We find that the Raman lasers indeed cause additional heating to the cloud. Nevertheless, the temperature we find is within the range of $0.5-0.8 T_{\mathrm{F}}$, which is still below degenerate temperature. In Figs. 2(d)-2(f), we also show $n_{\sigma}\left(k_{x}\right)-n_{\sigma}\left(-k_{x}\right)$ to reveal the distribution asymmetry more clearly.

Lifshitz transition.-With SO coupling, the single particle spectra of Eq. (1) are dramatically changed from two parabolic dispersions into two helicity branches as shown

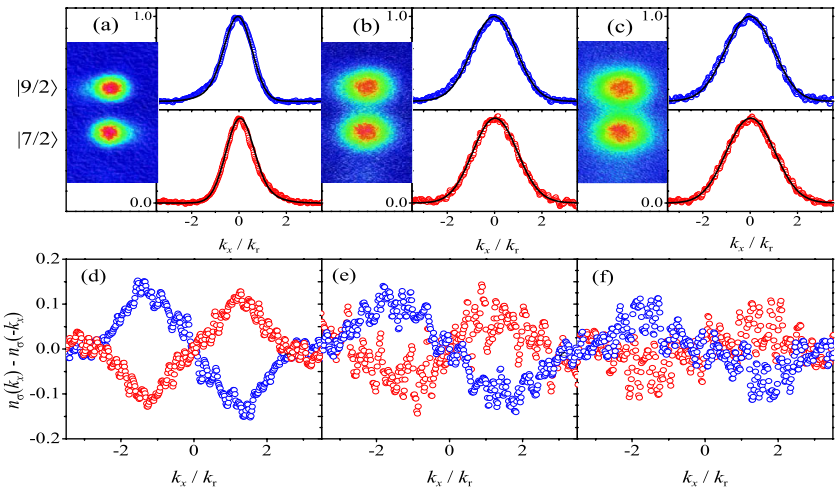

FIG. 2 (color online). Momentum distribution asymmetry as a hallmark of SO coupling: (a)-(c) time-of-flight measurement of momentum distribution for both $|\uparrow\rangle$ (blue) and $|\downarrow\rangle$ (red). Solid lines are theory curves. (a) $k_{\mathrm{F}}=0.9 k_{r}$ and $T / T_{\mathrm{F}}=0.8$; (b) $k_{\mathrm{F}}=1.6 k_{r}$ and $T / T_{\mathrm{F}}=0.63$; (c) $k_{\mathrm{F}}=1.8 k_{r}$ and $T / T_{\mathrm{F}}=$ 0.57. (d)-(f) Plot of integrated momentum distribution $n_{\sigma}(\mathbf{k})-$ $n_{\sigma}(-\mathbf{k})$ for the case of (a)-(c). in Fig. 3(b). Here, two different branches are eigenstates of "helicity" $\hat{s}$, and the helicity operator describes whether spin $\boldsymbol{\sigma}_{\mathbf{p}}$ is parallel or antiparallel to the "effective Zeeman field" $\mathbf{h}_{\mathbf{p}}=\left(-\Omega, 0, k_{\mathrm{r}} p_{x} / m+\delta\right)$ at each momentum, i.e., $\hat{s}=\boldsymbol{\sigma}_{\mathbf{p}} \cdot \mathbf{h}_{\mathbf{p}} /\left|\boldsymbol{\sigma}_{\mathbf{p}} \cdot \mathbf{h}_{\mathbf{p}}\right| . s=1$ for the upper branch, and $s=-1$ for the lower branch. The topology of the Fermi surface exhibits two transitions as the atom density varies. At sufficient low density, it contains two disjointed Fermi surfaces with $s=-1$, and they gradually merge into a single Fermi surface as the density increases to $n_{c 1}$. Finally, a new small Fermi surface appears at the center of the large Fermi surface when density further increases and fermions begin to occupy the $s=1$ helicity branch at $n_{c 2}$. A theoretical ground state phase diagram for the uniform system is shown in Fig. 3(a), and an illustration of the Fermi surfaces at different densities is shown in Fig. 3(b). Across the phase boundaries, the system experiences Lifshitz transitions as density increases [18], which is a unique property in a Fermi gas due to the Pauli principle.
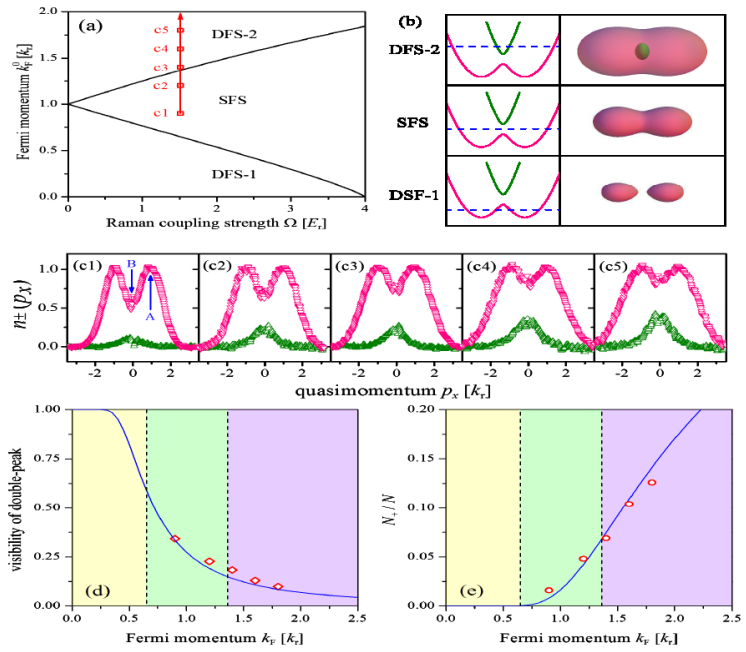

FIG. 3 (color online). Topological change of the Fermi surface and Lifshitz transition. (a) Theoretical phase diagram at $T=0$. $k_{\mathrm{F}}^{0}=\hbar\left(3 \pi^{2} n\right)^{1 / 3}$. "SFS" means single Fermi surface. "DFS" means double Fermi surface. (b) Illustration of different topologies of Fermi surfaces. The single particle energy dispersion is drawn for small $\Omega$. The dashed blue line is the chemical potential. (c) Quasimomentum distribution in the helicity bases. Red and green points are distributions for the $s=-1$ and $s=1$ helicity branches, respectively. $k_{\mathrm{F}}=0.9 k_{r}$ and $T / T_{\mathrm{F}}=0.80$ for (c1); $k_{\mathrm{F}}=1.2 k_{r}$ and $T / T_{\mathrm{F}}=0.69$ for $(\mathrm{c} 2) ; k_{\mathrm{F}}=1.4 k_{r}$ and $T / T_{\mathrm{F}}=0.61$ for $(\mathrm{c} 3) ; k_{\mathrm{F}}=1.6 k_{r}$ and $T / T_{\mathrm{F}}=0.63$ for $(\mathrm{c} 4)$; $k_{\mathrm{F}}=1.8 k_{r}$ and $T / T_{\mathrm{F}}=0.57$ for (c5). All these points are marked on the phase diagram in (a). (d) Visibility $v=\left(n_{\mathrm{A}}-n_{\mathrm{B}}\right) /$ $\left(n_{\mathrm{A}}+n_{\mathrm{B}}\right)$ decreases as $k_{\mathrm{F}} / k_{\mathrm{r}}$ increases [A and $\mathrm{B}$ points are marked in (c1)]. (e) Atom number population in $s=1$ helicity branch $N_{+} / N$ increases as $k_{\mathrm{F}} / k_{r}$ increases increases. In both (d) and (e), the blue solid line is a theoretical curve with $T / T_{\mathrm{F}}=$ 0.65 , and the background color indicates three different phases in the phase diagram. 
We fix the Raman coupling and vary the atom density at the center of the trap, as indicated by the red arrow in Fig. 3(a). The quasimomentum distribution in the helicity bases can be obtained from a transformation of momentum distribution in spin bases as follows [19]:

$$
\begin{aligned}
& n_{+}(\mathbf{p})=\frac{u_{\mathbf{p}}^{2} n_{\uparrow}\left(\mathbf{p}-k_{r} \hat{\mathbf{e}}_{x}\right)-v_{\mathbf{p}}^{2} n_{\downarrow}\left(\mathbf{p}+k_{r} \hat{\mathbf{e}}_{x}\right)}{u_{\mathbf{p}}^{2}-v_{\mathbf{p}}^{2}}, \\
& n_{-}(\mathbf{p})=\frac{v_{\mathbf{p}}^{2} n_{\uparrow}\left(\mathbf{p}-k_{r} \hat{\mathbf{e}}_{x}\right)-u_{\mathbf{p}}^{2} n_{\downarrow}\left(\mathbf{p}+k_{r} \hat{\mathbf{e}}_{x}\right)}{v_{\mathbf{p}}^{2}-u_{\mathbf{p}}^{2}} .
\end{aligned}
$$

In Fig. 3(c1-c5), we plot the quasimomentum distribution in the helicity bases for different atom densities. At the lowest density, the $s=1$ helicity branch is nearly unoccupied, which is consistent with the Fermi surface being below the $s=1$ helicity branch. The quasimomentum distribution of the $s=-1$ helicity branch exhibits clearly a double-peak structure, which reveals that the system is close to the boundary of having two disjointed Fermi surfaces at the $s=-1$ helicity branch. As density increases, the double-peak feature gradually disappears, indicating that the Fermi surface of $s=-1$ helicity branch finally becomes a single elongated one, as the top one in Fig. 3(b). Here we define a quality of visibility $v=\left(n_{\mathrm{A}}-n_{\mathrm{B}}\right) /$ $\left(n_{\mathrm{A}}+n_{\mathrm{B}}\right)$, where $n_{\mathrm{A}}$ is the density of the $s=-1$ branch at the peak and $n_{\mathrm{B}}$ is the density at the dip between two peaks. Theoretically, one expects that $v$ approaches unity at the low density regime and approaches zero at the high density regime. In Fig. 3(d), we show that our data decrease as density increases and agree very well with a theoretical curve with fixed temperature $T / T_{\mathrm{F}}=0.65$. Moreover, across the phase boundary between the single Fermi surface and double Fermi surface, one expects a significant increase of population on the $s=1$ helicity branch. In Fig. 3(e), the fraction of atom number population at the $s=1$ helicity branch is plotted as a function of Fermi momentum $k_{\mathrm{F}}$, which grows up nearby the critical point predicted in the zero-temperature phase diagram. The blue solid line is a theoretical calculation for $N_{+} / N$ with $T / T_{\mathrm{F}}=0.65$, and the small deviation between the data and this line is due to the temperature variation between different measurements. Because the temperature is so high that the transition is washed out, for both $v$ and $N_{+} / N$ we observe only a smooth decreasing or growth across the regime where it is supposed to have a sharp transition; however, the agreement with theory suggests that with better cooling a sharper transition should be observable.

Momentum-resolved rf spectroscopy.-The effect of SO coupling is further studied with momentum-resolved rf spectroscopy. The method is the same as first developed in Ref. [20]. Recently, spin injection spectroscopy has also been applied to study SO coupled Fermi gas by the Massachusetts Institute of Technology group [21]. A Gaussian shape pulse of the rf field is applied for $200 \mu \mathrm{s}$ to transfer atoms from the $|9 / 2,7 / 2\rangle(|\downarrow\rangle)$ state to the final

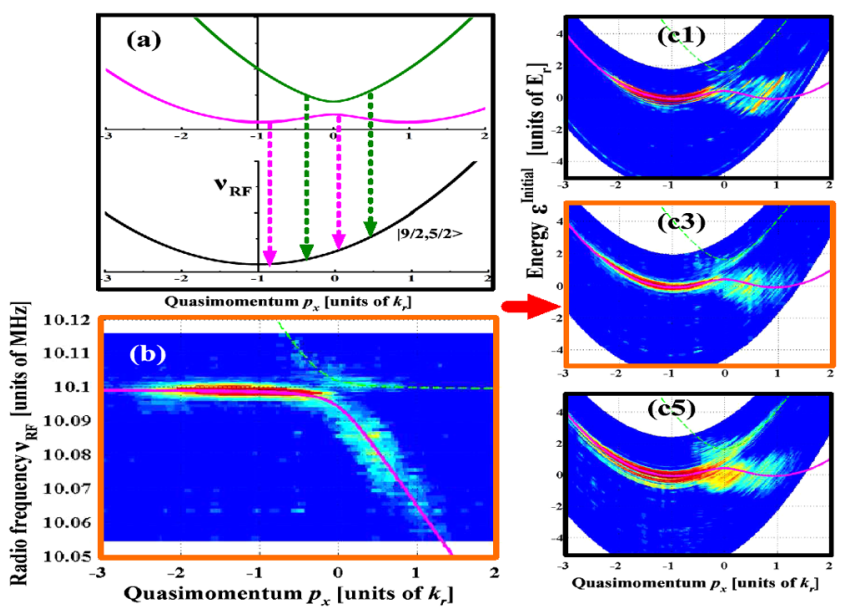

FIG. 4 (color online). Momentum-resolved rf spectroscopy of SO coupling Fermi gases. (a) Schematic of momentum-resolved rf spectroscopy of SO coupled Fermi gases. Green and pink solid lines are two helicity branches in which the eigenstates are all superpositions of $|9 / 2,9 / 2\rangle$ and $|9 / 2,7 / 2\rangle$. Thus both can undergo rf transition from $|9 / 2,7 / 2\rangle$ to $|9 / 2,5 / 2\rangle$, as indicated by dashed lines. (b) Intensity map of the atoms in the $|9 / 2,5 / 2\rangle$ state as a function of the $\left(\nu_{\mathrm{RF}}, k_{x}\right)$ plane. (c) Single particle dispersion and atom population measured for (c1), (c3), and (c5) in Fig. 3.

state $|9 / 2,5 / 2\rangle$, as shown in Fig. 4(a), and then the spin population at $|9 / 2,5 / 2\rangle$ is measured with time-of-flight at different rf frequencies. In Fig. 4(b), we plot an example of the final state population as a function of momentum $p_{x}$ and the frequency of rf field $\nu_{\mathrm{RF}}$, from which one can clearly see the backbending feature and the gap opening at the Dirac point. Both are clear evidence of SO coupling.

For an occupied state, the initial state dispersion $\epsilon(\mathbf{k})$ can be mapped out by

$$
\epsilon(\mathbf{k})=\hbar \nu_{\mathrm{RF}}-E_{Z}+\epsilon_{\mathrm{F}}(\mathbf{k}),
$$

where $\epsilon_{\mathrm{F}}(\mathbf{k})=\mathbf{k}^{2} / 2 m$ is the dispersion for the final $|9 / 2,5 / 2\rangle$ state and $E_{Z}$ is the energy difference between the $|9 / 2,7 / 2\rangle$ and $|9 / 2,5 / 2\rangle$ states. In Fig. 4(c), we show three measurements corresponding to (c1), (c3), and (c5) in Fig. 3. For (c1), clearly only the $s=-1$ branch is populated. For (c3), the population is slightly above the $s=1$ helicity branch. And for (c5), there is already significant population at the $s=1$ helicity branch. In Fig. 4(c5), one can also identify the chiral nature of two helicity branches. For the $s=-1$ branch, most left-moving states are dominated by the $|\downarrow\rangle$ state, while for the $s=1$ branch, right-moving states are mostly dominated by $|\downarrow\rangle$ states.

In summary, although the current temperature is still quite high due to the heating of the Raman laser and the current experiment is performed in the weakly interacting regime, we have still observed a few interesting phenomena of a SO coupled Fermi gas. In the near future, we will bring the system close to a Feshbach resonance where the $s$-wave interaction becomes strongly attractive, 
and we will further cool the system below the superfluid transition temperature. By confining the system into one dimension, we expect to find Majorana fermion modes at the phase boundaries when Fermi surface topology changes [22-24].

We thank Zhenhua Yu, Cheng Chin, Tin-Lun Ho, and Sandy Fetter for helpful discussions. This research is supported by National Basic Research Program of China (Grants No. 2011CB921601, No. 2010CB923103, and No. 2011CB921500), NSFC (Grants No. 10725416, No. 61121064, No. 11004118, and No. 11174176), DPFMEC (Approval No. 20111401130001), and Tsinghua University Initiative Scientific Research Program.

Note added.-Recently, we became aware of another experimental study of SO coupled Fermi gases using ${ }^{6} \mathrm{Li}[21]$.

*hzhai@mail.tsinghua.edu.cn jzhang74@sxu.edu.cn; jzhang74@yahoo.com

[1] Y.-J. Lin, K. Jiménez-García, and I. B. Spielman, Nature (London) 471, 83 (2011).

[2] T. D. Stanescu, B. Anderson, and V. Galitski, Phys. Rev. A 78, 023616 (2008).

[3] C. Wang, C. Gao, C.-M. Jian, and H. Zhai, Phys. Rev. Lett. 105, 160403 (2010).

[4] T.-L. Ho and S. Zhang, Phys. Rev. Lett. 107, 150403 (2011).

[5] C. J. Wu, I. Mondragon-Shem, and X. F. Zhou, Chin. Phys. Lett. 28, 097102 (2011).

[6] For a review, see M.Z. Hasan and C. L. Kane, Rev. Mod. Phys. 82, 3045 (2010).

[7] For a review, see also X. L. Qi and S. C. Zhang, Rev. Mod. Phys. 83, 1057 (2011).
[8] J.P. Vyasanakere and V.B. Shenoy, Phys. Rev. B 83, 094515 (2011).

[9] J. P. Vyasanakere, S. Zhang, and V. B. Shenoy, Phys. Rev. B 84, 014512 (2011).

[10] Z.-Q. Yu and H. Zhai, Phys. Rev. Lett. 107, 195305 (2011).

[11] H. Hu, L. Jiang, X.-J. Liu, and H. Pu, Phys. Rev. Lett. 107, 195304 (2011).

[12] M. Gong, S. Tewari, and C. Zhang, Phys. Rev. Lett. 107, 195303 (2011).

[13] K. Seo, L. Han, and C. A. R. Sa de Melo, arXiv:1201.0177.

[14] N. Goldman, I. Satija, P. Nikolic, A. Bermudez, M. A. Martin-Delgado, M. Lewenstein, and I. B. Spielman, Phys. Rev. Lett. 105, 255302 (2010).

[15] See Supplemental Material at http://link.aps.org/ supplemental/10.1103/PhysRevLett.109.095301 for more details.

[16] Z. Fu, P. Wang, S. Chai, L. Huang, and J. Zhang, Phys. Rev. A 84, 043609 (2011).

[17] Y.-J. Lin, R. L. Compton, A. R. Perry, W. D. Phillips, J. V. Porto, and I. B. Spielman, Phys. Rev. Lett. 102, 130401 (2009).

[18] I. M. Lifshitz, Sov. Phys. JETP 11, 1130 (1960).

[19] See Supplemental Material at http://link.aps.org/ supplemental/10.1103/PhysRevLett.109.095301 for the definition of $u_{\mathbf{p}}$ and $v_{\mathbf{p}}$.

[20] J. T. Stewart, J. P. Gaebler, and D. S. Jin, Nature (London) 454, 744 (2008).

[21] L. W. Cheuk, A. T. Sommer, Z. Hadzibabic, T. Yefsah, W. S. Bakr, M. W. Zwierlein, following Letter, Phys. Rev. Lett. 109, 095302 (2012).

[22] R. M. Lutchyn, J. D. Sau, and S. Das Sarma, Phys. Rev. Lett. 105, 077001 (2010).

[23] Y. Oreg, G. Refael, and F. von Oppen, Phys. Rev. Lett. 105, 177002 (2010).

[24] L. Jiang, T. Kitagawa, J. Alicea, A. R. Akhmerov, D. Pekker, G. Refael, J. I. Cirac, E. Demler, M. D. Lukin, and P. Zoller, Phys. Rev. Lett. 106, 220402 (2011). 